\title{
Energy Dissipation in the AFM Elasticity Measurements
}

\author{
O. Klymenko, J. Wiltowska-Zuber, M. LeKkA* and W.M. Kwiatek \\ Institute of Nuclear Physics \\ Radzikowskiego 152, 32-341 Cracow, Poland
}

\begin{abstract}
Nowadays, it is well established that changes of cell stiffness observed by atomic force microscopy are linked with the cell cytoskeleton. Its structural and functional alterations are underlying major diseases such as cancer, inflammation or neurodegenerative disorders. So far, the use of atomic force microscopy is mostly focused on the determination of the Young modulus using the modified Hertz model. It can quantitatively describe the elastic properties of living cells, however, its value is burdened by the fact that cells are neither isotropic nor homogeneous material. Often, during the atomic force microscopy measurements, the hysteresis between the loading and unloading curves are observed which indicates the dissipation of an energy. In our studies, the index of plasticity was introduced to enumerate such effect during a single loading-unloading cycle. As the results show, such approach delivers an additional parameter describing the mechanical state of cell cytoskeleton. The analysis was performed on test samples where the mechanical properties of the melanoma cells were changed by glutaraldehyde and cytochalasin D treatments. The non-treated cells were compared with fibroblasts.
\end{abstract}

PACS numbers: 68.37.Ps, 87.10.Vg, 87.15.La

\section{Introduction}

For many years, scientists have been employing many techniques to characterize the mechanical properties of normal and pathologically altered cells in their physiological conditions. In particular, the quantitative description of cancerous cells is of great interest since cancer transformation introduces significant changes in cell structure and behavior [1]. In particular, mechanical properties of the cell may be influenced, usually leading to higher cell deformability (i.e. lower Young's modulus) and to distinct adhesive interactions. Different deformability of cells is generally attributed to the altered cytoskeletal organization [2]. Low stiffness of cancer cells may be caused by a partial loss of actin filaments and/or microtubules, and therefore by the lower density of the cellular scaffold. Poor differentiation of the cell and the reduced adhesive interactions characterize a vast majority of cancer cells [3].

One of the methods enabling both qualitative and quantitative analysis of mechanical properties is the atomic force microscopy $(\mathrm{AFM})$. It is a high-resolution imaging technique that allows the quantitative measurements of mechanical properties of living cells in conditions close to physiological ones [4]. During past decades, AFM was applied to study the properties of biological samples ranging from single proteins to living cells. The stiffness of cells has been used to describe the role of cytoskeleton-associated proteins on the measured cell

* corresponding author; e-mail: Malgorzata.Lekka@ifj.edu.pl elasticity [5], the effect of drugs [6] or the influence of substrate properties [7]. So far, the use of AFM has been mostly focused on the determination of Young's modulus values using the modified Hertz model, which describes in a quantitative way the elastic properties of living cells [8]. However, its value is burdened by the fact that a cell is neither isotropic nor homogeneous material. Often, during the AFM measurements, the hysteresis between the loading and unloading curves is observed, indicating the dissipation of energy during the indentation measurement. Therefore, our studies were focused on the quantification of such hysteresis by the introduction of index of plasticity. The test samples (melanoma cells either softened using cytochalasin D or stiffened by glutaraldehyde fixation) were analyzed and their plasticity index was determined. Finally, the comparison of living melanoma cells with fibroblasts was performed. The results showed heterogeneity of viscoelastic-plastic properties of the cell. The different values of the plasticity index can be attributed to the distinct organization of actin filaments, and thereby it can describe the mechanical state of the cell cytoskeleton.

\section{Materials and the experimental method}

\subsection{Cell lines}

The AFM measurements were performed for two cell lines: human skin fibroblasts (CCL110, from LGC Promochem) and $1205 \mathrm{Lu}$ melanoma cells. The $1205 \mathrm{Lu}$ (ATCC CRL-2812) cell line was derived from lung metastases of WM793B cells after subcutaneous injection into immunodeficient mice. The chosen line is highly invasive and exhibits spontaneous metastasis to lung and liver [9]. 
Cells were cultured on glass coverslips in the RPMI 1640 medium $(\mathrm{pH} 7.4$ ) containing $10 \%$ foetal calf serum, at $37^{\circ} \mathrm{C}$ in a $95 \%$ air $/ 5 \% \mathrm{CO}_{2}$ incubator.

\subsection{Actin filaments depolymerization}

To depolymerize actin filaments, cells were incubated, with the $0.125 \mu \mathrm{M}$ solution of cytochalasin $\mathrm{D}$ dissolved in phosphate buffered saline (PBS, pH 7.4, containing $137 \mathrm{mM}$ of $\mathrm{NaCl}, 27 \mathrm{mM}$ of $\mathrm{KCl}$, Sigma), for $30 \mathrm{~min}$ at $37^{\circ} \mathrm{C}$ in a $95 \%$ air $/ 5 \% \mathrm{CO}_{2}$ incubator. Afterwards, cells were washed with PBS and immediately measured.

\subsection{Glutaraldehyde fixation}

Cells were fixed in $1 \%$ of glutaraldehyde (Fluka) dissolved in PBS for $5 \mathrm{~min}$, at $4^{\circ} \mathrm{C}$. Next, the coverslips with cells were washed with PBS and measured.

\subsection{Atomic force microscope}

The AFM, used for the measurements, was a home-built device, which was described in detail elsewhere [10]. It is equipped with a "liquid cell" setup made of plexiglass. All measurements were recorded at room temperature using commercial $\mathrm{Si}_{3} \mathrm{~N}_{4}$ cantilevers (MLCTAUHW; Veeco, Germany). The nominal spring constant of the cantilevers was $0.01 \mathrm{~N} / \mathrm{m}$.

Force curves were recorded in a square of about $7 \mu \mathrm{m} \times 7 \mu \mathrm{m}$ with the approach/retract velocity of $5.2 \mu \mathrm{m} / \mathrm{s}$. Four types of samples were measured: (i) living fibroblasts, (ii) living melanoma cells $1205 \mathrm{Lu}$, (iii) $1205 \mathrm{Lu}$ treated with cytochalasin $\mathrm{D}$, and (iv) $1205 \mathrm{Lu}$ fixed with glutaraldehyde. In total, 250-500 curves were analyzed for each sample type.

\subsection{Young's modulus determination}

Cell stiffness is usually determined on the basis of the force-versus-indentation curves obtained from the subtraction of deflections measured on stiff and compliant surfaces at given, relative sample position (Fig. 1a). Such curves describe the mechanical response to the applied loading force, which is characteristic of each material. The Young modulus value can be evaluated in the frame of the Hertz contact mechanics, taking into account an infinitely stiff indenter with a selected geometry [11]. Usually, the AFM probe tip is a four-sided pyramid that can be modelled either by cone or by paraboloid. The relationship between the loading force $F$ and the resulting indentation depth $\Delta z$ for conical shaped tip is following [11]:

$$
F(\Delta z)=\frac{2 E_{\text {cell }}(\Delta z)^{2}}{\pi \tan (\alpha)\left(1-\mu_{\text {cell }}^{2}\right)},
$$

where $\alpha$ is the opening angle of the cone, and $E_{\text {cell }}$ is the Young modulus, $\mu_{\text {cell }}$ is the Poisson ratio, related to the compressibility of the sample material. This value is set to be equal to 0.5 , since cells can be treated as incompressible material. The final Young modulus distribution is formed from values determined for all force curves recorded for a single cell.

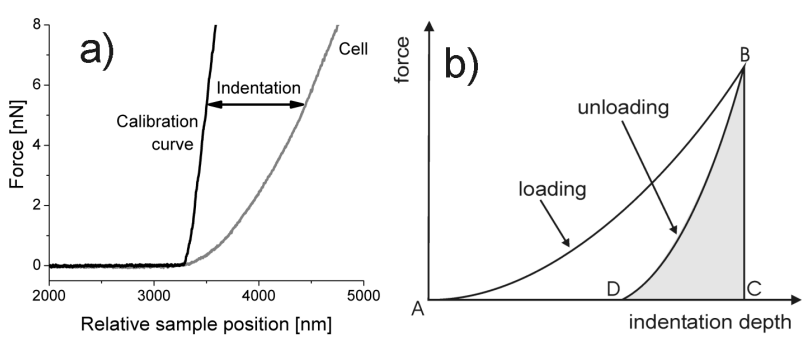

Fig. 1. (a) Determination of the force-versus-indentation curves. The black line corresponds to curves measured on hard, non-deformable surface (glass coverslip) and the nonlinear curve (gray line) - to compliant surface (cell). (b) The idea of the index of plasticity determination from a single loading/unloading indentation curve.

\subsection{Estimation of the energy dissipation}

Usually, for the Young modulus determination, only the part of force curve taken during approaching the sample surface is analyzed, assuming in addition that a living cell is an ideally elastic and isotropic material. Very often, the registered AFM loading/unloading curves show the significant hysteresis. To quantify the hysteresis, the plasticity index $\eta$ was introduced [12]. In a solid body, it is usually a parameter which characterizes the relative plastic/elastic behavior of the material when it undergoes external force. In the indentation measurements, its value can be determined from the ratio between areas under loading and unloading curves $\left(\eta=1-A_{2} / A_{1}\right.$, where $A_{1}$ and $A_{2}$ are areas under loading and unloading curves, i.e. regions $A B C$ and $D B C$ in Fig. 1b, respectively). The index of plasticity covers the range between $\eta=0$, when $A_{2}=A_{1}$ i.e. when loading and unloading overlap (fully elastic behavior), and $\eta=1$ when $A_{2}=0$ (fully plastic), while the intermediate values $0<\eta<1$ indicate mixed viscoelastic-plastic properties.

\section{Results and discussion}

\subsection{Young's modulus of living cells}

Figure 2a and $\mathrm{b}$ presents the histogram of the Young modulus determined for melanoma cells and fibroblasts. The obtained mean Young modulus values were of $0.7 \pm 0.5 \mathrm{kPa}$ and $1.2 \pm 0.4 \mathrm{kPa}$, correspondingly (data calculated from Gaussian fit, for the indentation depth of $500 \mathrm{~nm}$ ). Cell indentation generates the response from inner structural cell components [13]. For small indentations depths the values of the modulus are dominated by the mechanical properties of the cell membrane. Larger deformations include the propeties of actin filaments underlying beneath cell membrane, which enables to probe in a quantitative way their organization inside the cell. The comparison of dimensions of all interacting objects and the indentation depth value $(500 \mathrm{~nm})$ suggests that the response of the cellular scaffold is the most important in the presented measurements. The obtained Young 
modulus values pointed out similar mechanical properties of actin filaments in both cell types with a slight tendency for cancerous melanoma cells to be more deformable than fibroblasts.
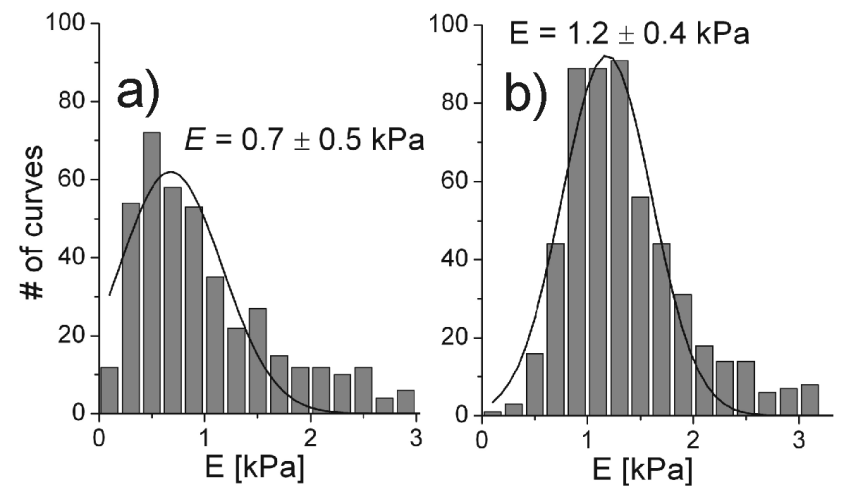

Fig. 2. Histograms of the Young modulus for living cells (a) melanoma (1205Lu) and (b) fibroblasts.

The large experimental error originates from uncertainties in the tip shape, the spring constant, the contact area, sample heterogeneity, and approximations in the theoretical model used for analysis.

\subsection{Index of plasticity of cytochalasin D-treated and fixed cells}

Along with the Young modulus value, one can also determine the index of plasticity $\eta$ (as described in Sect. 2.6) that provides the information about local, viscoelastic-plastic properties of the cell surface with regard to the contribution of the cell elasticity. Therefore, the value of $\eta$ was determined for $1205 \mathrm{Lu}$ melanoma cells (Fig. 3a and b), which were either softened or stiffened. The cell softening was induced by the cytochalasin D $\left(0.125 \mu \mathrm{M}\right.$ for $30 \mathrm{~min}$ at $\left.37^{\circ} \mathrm{C}\right)$, whereas the application of glutaraldehyde $\left(1 \%\right.$, for $5 \mathrm{~min}$ at $\left.4^{\circ} \mathrm{C}\right)$ resulted in cells stiffening. The obtained histograms of the plasticity index clearly showed few maxima regardless of the cell treatment, thus reflecting the heterogeneity of the cell surface. In addition, the discrete character was clearly observed. In case of cytochalasin $\mathrm{D}$ treatment, three maxima of $\eta$ value were present, centered at $0.67 \pm 0.04$, $0.80 \pm 0.04$, and $0.88 \pm 0.04$, while those for glutaraldehyde fixed cells were positioned at $0.02 \pm 0.05$ and $0.18 \pm 0.01$. The incubation with cytochalasin $\mathrm{D}$ leads to depolymerization of actin filaments $[14,15]$ and thus, after the indentation of the cell, they cannot quickly come back to initial (pre-indentation) conditions (the larger values of the plasticity index are expected). The lack of contribution of viscoelastic-plastic properties after glutaraldehyde fixation, most probably, was connected with the stiffening of the cell. The applied maximum value of the loading force could not induce the energy dissipation.

The plasticity index was also determined for living cells (Fig. 3c and d). Similarly to the treated cells,
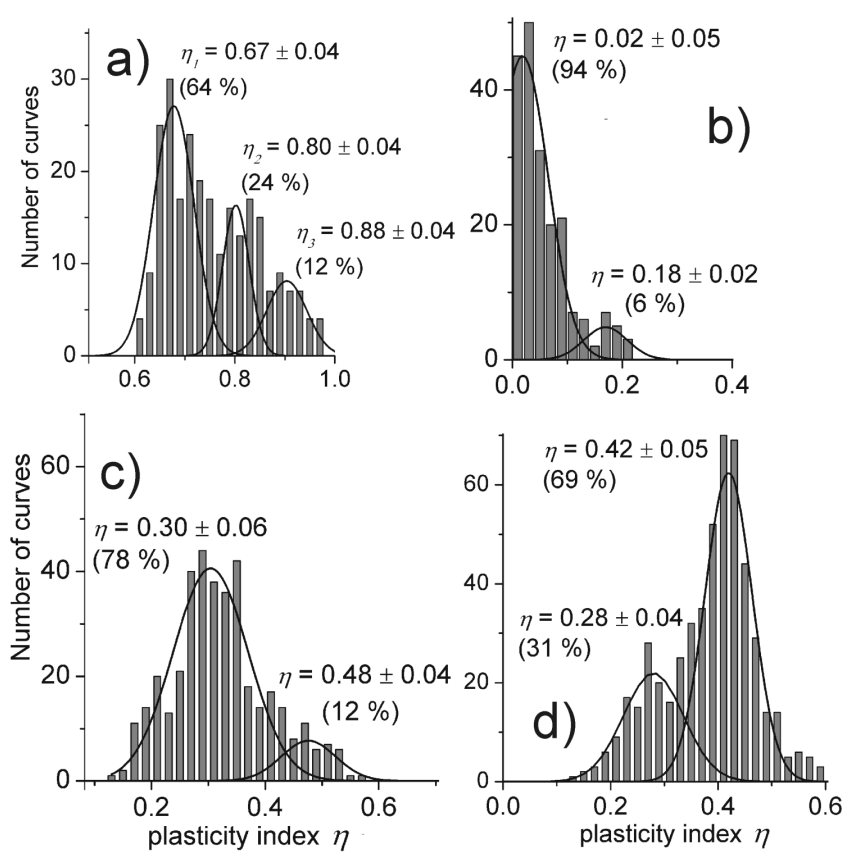

Fig. 3. Distributions of the plasticity index for melanoma cells $(1205 \mathrm{Lu})$ treated with (a) $0.125 \mu \mathrm{M}$ cytochalasin D and (b) $1 \%$ glutaraldehyde. The plasticity index for (c) melanoma cells and (d) fibroblasts was determined for living cells measured in physiological conditions. The percentage in parentheses denotes the contribution of each maximum, estimated taking into account the area under each peak.

two maxima are observed in the histograms. Each of them corresponds to the loading/unloading curve showing more viscoelastic-plastic $(0.48 \pm 0.04$ and $0.42 \pm 0.05$, for melanoma and fibroblasts, respectively) or more elastic behavior. For the latter one, the index of plasticity for both cell types was very similar $(0.30 \pm 0.06$ and $0.28 \pm 0.04)$.

The living cell properties showed the intermediate values and still the discrete character was visible. Also, fibroblasts showed slightly higher values of plasticity index than melanoma cells. The values of the plasticity index close to 1 strongly suggest that the disruption of the cytoskeleton leads to the state when energy is dissipated during indentation experiments. Moreover, the discrete character of $\eta$ implies the heterogeneity of cell surface, pointing out locations with large or small energy dissipation. Values of the plasticity index near 0 indicate behavior close to fully elastic material where there is no energy dissipation. This case has been observed for cells fixed with glutaraldehyde. Despite the similar $\eta$ values determined from the histograms (Fig. 3c and d), the prominent difference in the population of measured curves was observed. For melanoma cells, only $12 \%$ of curves showed more plastic behavior while the rest of curves present larger elastic contribution. The fibroblasts behaved oppositely - $69 \%$ of recorded force curves demonstrate large plasticity contribution while only $31 \%$ show the domination of the elasticity. 


\section{Conclusions}

So far the determination of the index of plasticity has been applied to characterize mainly polymer surfaces [12]. Our results showed that this approach can be reasonably applied to the description of the viscoelastic-plastic properties of living cells. Such methodology enables to analyze and to quantify the energy dissipation during the single AFM indentation measurement where loading/unloading curves are recorded.

Independently of the cell type (melanoma cells or fibroblasts) or of their treatment (glutaraldehyde fixation or depolymerization of actin filaments by cytochalasin D), two (for $1205 \mathrm{Lu}$ melanoma cells three) discrete values of the plasticity index $\eta$ were obtained. The smaller $\eta$ values correspond to locations revealing the properties of the elastic material while the larger ones can be ascribed to the position where the energy dissipation occurred.

Measurements performed on cells stiffened by glutaraldehyde fixation bring the $\eta$ values close to 0 representing the elastic response, while cells softened by cytochalasin $\mathrm{D}$ showed large $\eta$ values within the range of $0.7-0.9$. This value indicates the dominant contribution from viscoelastic-plastic properties of cells.

The plasticity index of melanoma cells and fibroblasts, measured for living cells in a physiological buffer, was in the same range, from 0.2 to 0.5 . However, the relation between the elastic and viscoelastic fractions in these two cell types was reversed (cf. Fig. 3c, d). Its variations and the shape of the distribution can be attributed to distinct and heterogeneous properties of actin cytoskeleton composed of filaments lying beneath cell membrane. Thus, the plasticity index can be used as an additional quantity describing the mechanical state of cell cytoskeleton. These preliminary results demonstrate the feasibility of the method, however, further most systematic studies are required.

\section{References}

[1] D. Hanahan, R.A. Weinberg, Cell 100, 57 (2000).

[2] S. Suresh, Acta Biomater. 3, 413 (2007).

[3] A. Ben-Ze'ev, Curr. Opin. Cell Biol. 9, 99 (1997).

[4] P. Parot, Y.F. Dufrene, P. Hinterdorfer, C. Le Grimellec, D. Navajas, J.L. Pellequer, S. Scheuring, J. Mol. Recognit. 20, 418 (2007).

[5] R.M. Ezzell, W.H. Goldmann, N. Wang, N. Parasharama, D.E. Ingber, Exp. Cell Res. 231, 14 (1997).

[6] M. Lekka, P. Laidler, J. Ignacak, M. Łabẹdź, J. Lekki, H. Struszczyk, Z. Stachura, A.Z. Hrynkiewicz, Biochim. Biophys. Acta 1540, 127 (2001).

[7] J. Domke, S. Dannohl, W.J. Parak, O. Mueller, W.K. Aicher, M. Radmacher, Colloids Surf. B 19, 367 (2000).

[8] M. Radmacher, IEEE Eng. Med. Biol. Magn. 16, 47 (1997).

[9] I. Juhasz, S.M. Albelda, D.E. Elder, G.F. Murphy, K. Adachi, D. Herlyn, I.T. Valyi-Nagy, M. Herlyn, Am. J. Pathol. 143, 528 (1993).

[10] M. Lekka, J. Lekki, M. Marszałek, P. Golonka, Z. Stachura, B. Cleff, A.Z. Hrynkiewicz, Appl. Surf. Sci. 141, 345 (2000).

[11] I.N. Sneddon, Int. J. Eng. Sci. 3, 47 (1965).

[12] B.J. Briscoe, L. Fiori, E. Pelillo, J. Phys. D, Appl. Phys. 31, 2395 (1998).

[13] S. Kasas, G. Dietler, Pflugers Arch. 456, 13 (2008).

[14] M. Schliwa, J. Cell Biol. 92, 79 (1982).

[15] H.W. Wu, T. Kuhn, V.T. Moy, Scanning 20, 389 (1998). 